\title{
MicroRNA let-7a suppresses the growth and invasion of cholesteatoma keratinocytes
}

\author{
WENJING ZHANG ${ }^{*}$, XIAOHUA CHEN ${ }^{*}$ and ZHAOBING QIN \\ Department of Otology, The First Affiliated Hospital of Zhengzhou University, \\ Zhengzhou, Henan 450003, P.R. China
}

Received January 7, 2014; Accepted October 24, 2014

DOI: $10.3892 / \mathrm{mmr} .2014 .2971$

\begin{abstract}
Cholesteatomas are benign epidermally-derived lesions of the temporal bone that are caused by migration of hyperproliferative keratinocytes into the middle ear and mastoid cavity. The molecular mechanisms that regulate the pathogenesis of cholesteatomas are currently not fully understood. The present study demonstrated the antigrowth and anti-invasive effects of let-7a microRNA (miRNA) on cholesteatoma keratinocytes. Let-7a inhibited the growth of cholesteatoma keratinocytes through two different mechanisms: Restriction of the proliferation of keratinocytes by promoting cell cycle arrest in the G0/G1 phase, and the induction of apoptosis of the cells. In addition to its role in the inhibition of cell growth, let-7a suppressed the migration and invasion of cholesteatoma keratinocytes. A mechanistic study showed that let-7a downregulated the expression of miR-21. Considering the function of miR-21 in the regulation of proliferation and apoptosis, let-7a may control cell proliferation and apoptosis by regulating miR-21, and its targets, in cholesteatoma keratinocytes. In conclusion, the present study showed that let-7a downregulates the expression of miR-21, resulting in the suppression of proliferation and induction of apoptosis. The results of the present study reveal the crucial role of let-7a miRNA in the inhibition of growth and invasion of cholesteatoma keratinocytes.
\end{abstract}

\section{Introduction}

Cholesteatomas are gradually expanding, destructive lesions of the temporal bone (1). The presence of destructive epithelial lesions of the temporal bone results in the erosion of adjacent

Correspondence to: Dr Zhaobing Qin, Department of Otology, The First Affiliated Hospital of Zhengzhou University, 1 Jianshe Road, Zhengzhou, Henan 450003, P.R. China

E-mail: zhaobing.qin@hotmail.com

*Contributed equally

Key words: let-7a, cholesteatoma, keratinocyte, microRNA, miR-21 bone structures, and leads to various complications, including otalgia, malodorous otorrhea and hearing loss (2). There are two types of cholesteatomas: Congenital and acquired (2). The common feature of these two types of cholesteatomas is the migration of keratinized hyperproliferative squamous epithelium, from a fibrous stroma into the middle ear and mastoid cavity (2). A previous study demonstrated that keratinocyte proliferation and migration is mediated by growth factors and their receptors (1). An upregulation of epidermal growth factor (EGF) and its receptor (EGFR), and of keratinocyte growth factor (KGF) and its receptor (KGFR) have previously been reported in cholesteatomas (3-6). Further studies have suggested that an upregulation of these growth factors and their receptors induces cell proliferation of keratinocytes in cholesteatomas (7-9). In addition to growth factors, the possible roles of microRNAs (miRNA) in the formation of cholesteatomas have recently been proposed $(10,11)$.

miRNAs are evolutionarily conserved, small non-coding RNA molecules, and are considered to be important post-transcriptional modifiers (12). miRNAs have significant roles in the regulation of cellular proliferation, differentiation, apoptosis and oncogenesis (13). Previous studies have shown that miRNAs are strongly associated with the development of cholesteatomas $(10,11)$. Friedland et al $(11)$, demonstrated an upregulation of human miR-21, and a reduction of its targets, phosphatase and tensin homologue (PTEN) and programmed cell death protein (PDCD4) in cholesteatomas. In our previous study, it was shown that the levels of miR-21 and let-7a miRNA were significantly increased, particularly in pediatric patients (10). Furthermore, the expression levels of PTEN and PDCD4 were decreased in cholesteatoma tissues (10). These findings support the potential roles of miR-21 and let-7a miRNA in the pathogenesis of cholesteatomas.

The present study aimed to investigate the functions of let $7 \mathrm{a}$ in cholesteatoma keratinocytes using let $7 \mathrm{a}$ miRNA mimics and a let $7 \mathrm{a}$ inhibitor. In particular, the role of let- $7 \mathrm{a}$ miRNA on cellular proliferation, apoptosis and migration in cholesteatoma keratinocytes was evaluated.

\section{Materials and methods}

Tissue collection. The present study was approved by the Ethics Committee of the First Affiliated Hospital of Zhengzhou University (Zhengzhou, China). Written informed 
consent was obtained from all of the patients, or from the parents of the patients, prior to surgery. Cholesteatoma tissues were collected from 20 cholesteatoma patients (aged 18-70 years), and normal postauricular skin specimens from the same patients served as the control tissues. The samples were transported to the laboratory immediately following surgery.

Cell culture and miRNA transfection. Cholesteatoma tissues were transferred into $5 \mathrm{ml}$ keratinocyte free media (KSFM; Gibco-BRL, Carlsbad, CA, USA). The tissues were then treated with $0.5 \mathrm{ml}$ collagenase $(20 \mathrm{mg} / \mathrm{ml})$, and dissociated into single cells. The suspension was centrifuged for $5 \mathrm{~min}$ at 287 x g (Thermo Labofuge 400R; Thermo Fisher Scientific, Waltham, MA, USA). The pellets were resuspended in $10 \mathrm{ml}$ KSFM supplemented with penicillin and streptomycin (Sigma-Aldrich, Santa Clara, CA, USA, 10ml/1 vol/vol). The cells were cultured in a humidified incubator containing $5 \%$ $\mathrm{CO}_{2}$ at $37^{\circ} \mathrm{C}$. The mimics, inhibitor and control miRNA were purchased from GenePharma, Co., Ltd., Shanghai, China. Cells were transfected with let $7 \mathrm{a}$ inhibitor, let $7 \mathrm{a}$ mimics or a negative control miRNA according to the manufacturer's instructions. In brief, $2.5 \mu 1$ Lipofectamine ${ }^{\circledR} 2000$ (Invitrogen Life Technologies, Carlsbad, CA, USA) was mixed with $50 \mu 1$ serum-free medium in a sterile tube. Following incubation for $15 \mathrm{~min}, 15$ pmol mimic, inhibitor or control miRNA was added to the mixture for a further $15 \mathrm{~min}$. The mixture was subsequently added to the 24-well plates and total RNA was extracted on the third day following transfection.

Reverse transcription-quantitative polymerase chain reaction (RT-qPCR). Cholesteatoma tissues from each surgical specimen ( $30 \mathrm{mg})$ were homogenized using a syringe and needle. High-molecular-weight DNA may be sheared by passing the lysate through a 20-gauge needle, attached to a sterile plastic syringe, at $\geq 5-10$ times until a homogeneous lysate was achieved. Increasing the volume of lysis buffer may be required to facilitate handling and minimize sample loss. Cells were lysed using TRIzol ${ }^{\circledR}$ reagent (Invitrogen Life Technologies), according to the manufacturer's instructions. Total miRNAs were extracted with the miRNeasy kit (GenePharma, Co., Ltd., Shanghai, China). qPCR was performed using a 7500 Fast Real-time PCR system (Applied Biosystems Life Technologies, Foster City, CA, USA). Briefly, $1 \mu \mathrm{g}$ total RNA was reverse-transcribed into cDNA using a kit purchased from GenePharma Co., Inc., and amplified by qPCR using gene-specific primers. The sequences of the primers used were as follows: Forward: 5'-ACGTTGTGTAGCTTATCAGACTG-3' and reverse: 5'-AATGGTTGTTCTCCACACTCTC-3' for miR-21; forward: 5'-CGATTCAGTGAGGTAGTAGGTTGT-3' and reverse: 5'-TATGGTTGTTCTGCTCTCTGTCTC-3' for let-7a; and forward: 5'-ATTGGAACGATACAGAGAAGATT-3' and reverse: 5'-GGAACGCTTCACGAATTTG-3' for U6. The PCR conditions were as follows: $95^{\circ} \mathrm{C}$ for 3 minutes, followed by 40 cycles of $95^{\circ} \mathrm{C}$ for $12 \mathrm{sec}$ and $62^{\circ} \mathrm{C}$ for $40-60$ seconds. For all of the reactions, no-template controls and random RNA preparations were also subjected to reverse transcription, in order to verify the absence of genomic DNA amplification. The relative miRNA expression levels were calculated using the $2^{-\Delta \Delta \mathrm{CT}}$ method.
Cell cycle analysis. For cell cycle analysis, the control and transfected cells were washed with phosphate-buffered saline (PBS; Gibco-BRL), fixed with $90 \%$ ethanol overnight at $4^{\circ} \mathrm{C}$ and incubated with RNase (Sigma-Aldrich) at $37^{\circ} \mathrm{C}$ for $30 \mathrm{~min}$. The nuclei of the cells were stained with propidium iodide (PI) for an additional $30 \mathrm{~min}$. A total of $10^{4}$ nuclei were examined in a FACSCalibur ${ }^{\mathrm{TM}}$ flow cytometer (BD Biosciences, San Jose, CA, USA). The experiments were performed in triplicate. The results were presented as a percentage of the cells in a particular phase.

5-Ethynyl-2'-deoxyuridine (EdU) incorporation assay. A total of $10 \mu \mathrm{M}$ EdU (Cat. no. C10310; Ribobio, Co., Ltd., Guangzhou, China) was added to the cultured cells for $24 \mathrm{~h}$. The cells were then washed with PBS and fixed with $4 \%$ paraformaldehyde (Solarbio, Beijing, China). The cells were incubated with $2 \mathrm{mg} / \mathrm{ml}$ glycine and washed with PBS twice. Following permeabilization with PBS containing $0.5 \%$ Triton X-100 (Sigma-Aldrich) and extensive washing with PBS, the cells were incubated with Apollo ${ }^{\circledR}$ staining solution (Guangzhou Ribo Bio, Guangzhou, China) for $30 \mathrm{~min}$. The cells were washed a further three times with PBS containing $0.5 \%$ Triton X-100, and then incubated with Hoechst 33342 (Sigma-Aldrich) for $10 \mathrm{~min}$. The images of the staining were captured using an Olympus BX43 fluorescent microscope (Olympus Corp., Tokyo, Japan).

Annexin V-fluorescein isothiocyanate (FITC) apoptosis assay. To quantify let-7a miRNA-induced apoptosis, Annexin V/propidium iodide and phycoerythrin (PIPE) staining was performed. The apoptotic rate of the cells was evaluated by flow cytometric analysis. Briefly, the cells were treated with the let-7a inhibitor, let-7a mimics or the negative control miRNA. The treated cells were collected and subjected to Annexin V/PI staining using an Annexin V-FITC Apoptosis Detection kit (BioVision, Inc., Milpitas, CA, USA), according to the manufacturer's instructions. The fluorescence was measured by fluorescence-activated cell sorting (FACSCalibur; BD Biosciences).

Terminal deoxynucleotidyl transferase-mediated dUTP-digoxigenin nick end-labeling (TUNEL) staining. The apoptosis of the cholesteatoma keratinocytes transfected with the let-7a inhibitor, let-7a mimics or negative control miRNA was detected using a TUNEL kit (Roche, Shanghai, China). Briefly, the cells were incubated with terminal deoxynucleotidyl transferase enzyme solution for $60 \mathrm{~min}$, washed twice with PBS and incubated for $30 \mathrm{~min}$ with 4',6-diamidino-2-phenylindole (Yeasen, Shanghai, China). The images of the staining were captured using a fluorescent microscope.

Cell invasion assay. The invasive abilities of the cells were examined using 6-well Transwell plates (Corning, Inc., New York, NY, USA). The cells transfected with let-7a inhibitor, let-7a mimics or negative control miRNA were removed from the culture flasks and resuspended in serum-free medium $\left(5 \times 10^{5}\right.$ cells $\left./ \mathrm{ml}\right)$. A total of $200 \mu \mathrm{l}$ of each cell suspension was added to the upper chambers of Transwell plates. The chambers were incubated for $48 \mathrm{~h}$ at 
$37^{\circ} \mathrm{C}$. The filters were then stained with hexamethylpararosaniline staining solution (Yeasen). The upper surfaces of the filters were scraped twice with cotton swabs to remove non-migrated cells. The experiment was repeated three times, and the migrated cells were counted in five different fields per filter.

Statistical analysis. All statistical analyses were performed using SPSS statistical software, version 17.0 (SPSS, Inc., Chicago, IL, USA). Statistical significance was determined by two-tailed Student's t-test. $\mathrm{P}<0.05$ was considered to indicate a statistically significant difference. All experiments were repeated $\geq 3$ times and the data are expressed as the mean \pm standard deviation.

\section{Results}

Let-7a miRNA suppresses growth by inhibiting cell proliferation of cholesteatoma keratinocytes. To investigate the function of let-7a during the development of cholesteatoma keratinocytes, the cell number of miRNA-transfected cholesteatoma keratinocytes was compared (Fig. 1). The cell number was reduced when the cells were transfected with mimics of let-7a (Fig. 1A). Whereas, the cell number was increased when the cells were transfected with the inhibitor of let-7a (Fig. 1A). These data suggest that let-7a may restrict the growth of cholesteatoma keratinocytes. An EdU incorporation assay was used to determine whether let-7a impacts the proliferation of cholesteatoma keratinocytes. The number of proliferative EdU-positive $\left(\mathrm{EdU}^{+}\right)$cells increased following transfection with the let-7a inhibitor, as compared with the miRNA control (Fig. 1Ba and Bg). Whereas, the number of $\mathrm{EdU}^{+}$cells was decreased following transfection with let-7a mimics (Fig. 1Bd and Bg). The number of EdU ${ }^{+}$cells was significantly increased following transfection with the let-7a inhibitor, and significantly decreased following transfection with let-7a mimics (Fig. 1C).

To confirm that let-7a inhibited cellular proliferation by regulating the cell cycle progression of the cholesteatoma keratinocytes, cell cycle analysis was performed. The distribution of the cells in the different phases of the cell cycle was examined by FACS, $48 \mathrm{~h}$ post-transfection with the various miRNAs. The percentage of cells in the $S$ phase increased from $26.90 \%$ in the control group (Fig. 2A), to $39.33 \%$ in the group transfected with the let-7a inhibitor (Fig. 2B). This percentage was reduced to $15.23 \%$ in the group transfected with let-7a mimics (Fig. 2C). Concordantly, the percentage of cells in the $\mathrm{G} 2 / \mathrm{M}$ phase increased from $19.61 \%$ in the control group (Fig. 2A), to $20.28 \%$ in the group transfected with the let-7a inhibitor (Fig. 2B). The percentage of cells in the G2/M phase was reduced to $16.29 \%$ in the group transfected with let-7a mimics (Fig. 2C), as compared with the control group. Conversely, the percentage of cells in the G0/G1 phase was reduced from $53.49 \%$ in the control group (Fig. 2A), to $41.39 \%$ following transfection with the let-7a inhibitor (Fig. 2B). The percentage of cells in the G0/G1 phase was increased to $68.48 \%$ following transfection with the let-7a mimics (Fig. 2C), as compared with the control. These results suggest that let-7a promotes the arrest of cholesteatoma keratinocytes at the $\mathrm{G} 0 / \mathrm{G} 1$ phase.
These data demonstrate that let-7a suppresses proliferation of keratinocytes, by promoting cell cycle arrest in the G0/G1 phase.

Let-7a miRNA induces cell apoptosis of cholesteatoma keratinocytes. To explore whether let-7a impacts cell growth by regulating apoptosis, early apoptosis was examined in the cholesteatoma keratinocytes using an Annexin V-FITC Apoptosis Detection kit. The percentage of cells displaying the features of early apoptosis was elevated from $17.32 \%$ in the control cells (Fig. 3A), to $28.96 \%$ in the cells transfected with let-7a mimics (Fig. 3B). Conversely, this number was reduced to $4.55 \%$ in the cells transfected with the let- $7 \mathrm{a}$ inhibitor (Fig. 3C). The percentage of early apoptotic cells was significantly decreased in the cells transfected with the let-7a inhibitor, as compared with the cells transfected with the control miRNA (Fig. 3D). Whereas, the percentage was significantly increased in the cells transfected with let-7a mimics (Fig. 3D).

To confirm the effects of let-7a on cell apoptosis, the apoptotic rate of the cholesteatoma keratinocytes transfected with the different miRNAs were examined using TUNEL. The number of TUNEL-positive cells was decreased in the cholesteatoma keratinocytes transfected with the let-7a inhibitor and increased in the keratinocytes transfected with let-7a mimics (Fig. 4A). The percentage of apoptotic cells following transfection with the let-7a inhibitor or let-7a mimics was significantly decreased and increased, respectively (Fig. 4B). These results suggest that let-7a induces cell apoptosis of cholesteatoma keratinocytes.

Let-7a miRNA inhibits cell invasion of cholesteatoma keratinocytes. An aim of the present study was to determine whether let-7a affects cell invasion of cholesteatoma keratinocytes. Therefore, Matrigel ${ }^{\mathrm{TM}}$ invasion assays were performed using $8.0 \mu \mathrm{m}$ pore-size Transwell plates, which allow cell migration across the filter. The number of cholesteatoma keratinocytes that migrated across the Matrigel ${ }^{\mathrm{TM}}$ and the insert was significantly altered following transfection with the various miRNAs (Fig. 5A-C). The percentage of migrated cells transfected with the let-7a inhibitor was 1.75 times higher, as compared with the cells transfected with the negative control miRNA $(92.3 \pm 5.7$ vs $52.7 \pm 6.0 ; \mathrm{P}<0.05)$ and 4.87 times higher as compared with those transfected with let-7a mimics $(92.3 \pm 5.7$ vs $18.9 \pm 5.3$; $\mathrm{P}<0.05)$. These results indicate that let-7a prevents invasion and migration of cholesteatoma keratinocytes.

Let-7a miRNA affects the expression of miR-21 in cholesteatoma keratinocytes. Considering the important roles of let-7a in the pathogenesis of cholesteatomas, it may be beneficial to identify the downstream targets regulated by let-7a. Previous studies demonstrated that high expression levels of miR-21 in cholesteatoma tissues regulated cell proliferation and apoptosis $(10,11)$. Therefore it may be hypothesized that let-7a regulates the expression of miR-21. To determine a potential role for let-7a in the regulation of miR-21, the effects of the let-7a inhibitor and mimics were assessed on miR-21 expression in cholesteatoma keratinocytes. There was a downregulation in the expression levels of miR-21 in the cholesteatoma keratinocytes transfected with the let-7a 

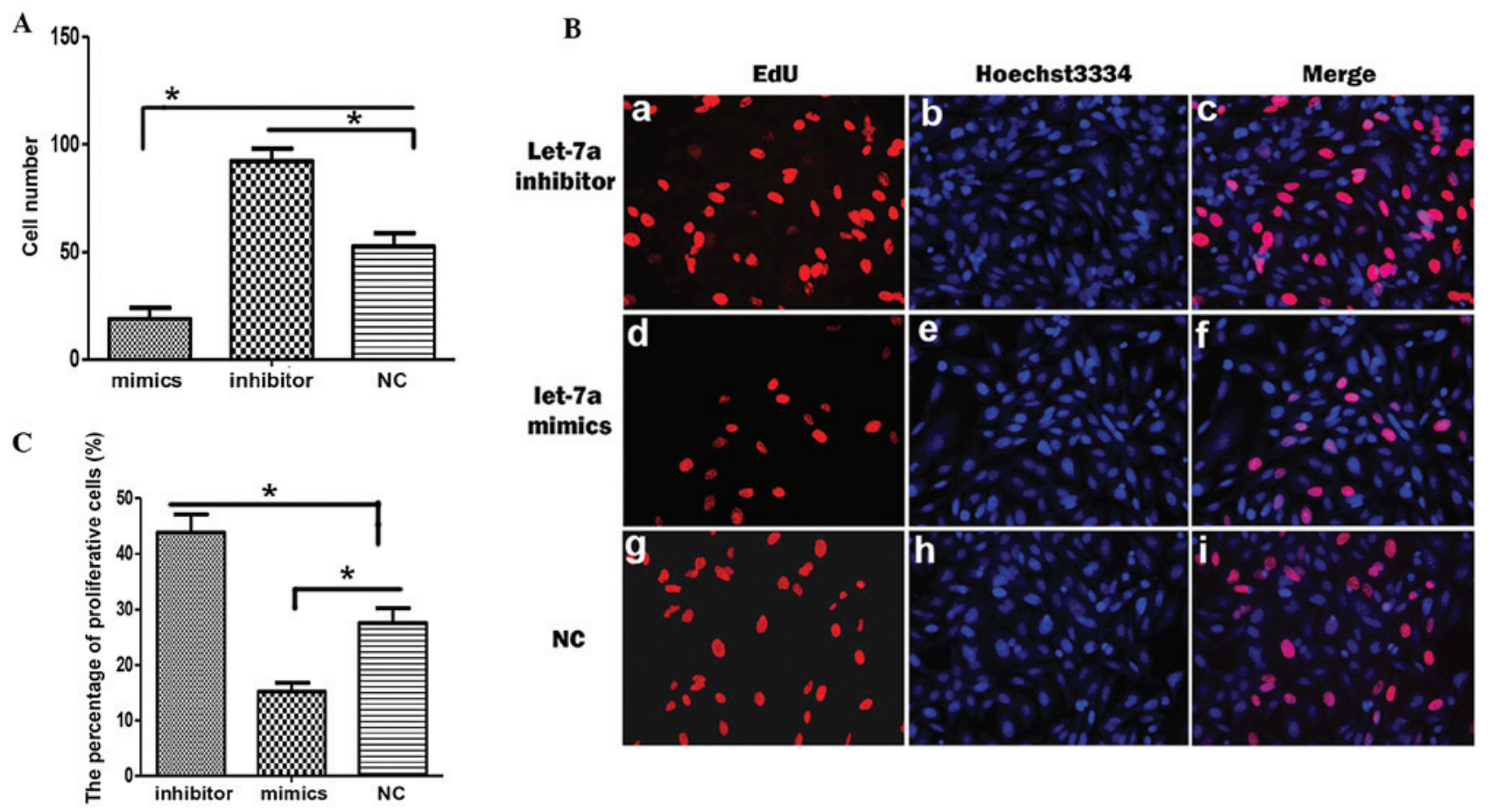

Figure 1. Let-7a inhibits proliferation of cholesteatoma keratinocytes. (A) The number of cholesteatoma keratinocytes was counted following transfection with let-7a mimics, let-7a inhibitor or control miRNA (NC). (B) The representative images of 5-ethynyl-2'-deoxyuridine (EdU) immunostaining (red; a, d, g), Hoechst 3334 staining (blue; b, e, h) and their merged images (c, f, i) in cholesteatoma keratinocytes transfected with a let-7a inhibitor, let-7a mimics or NC. (C) Statistical analysis of the percentage of proliferative cells following transfection with a let-7a inhibitor, let-7a mimics or NC. Data are represented as the mean \pm standard deviation. ${ }^{*} \mathrm{P}<0.05$, Student's t-test.
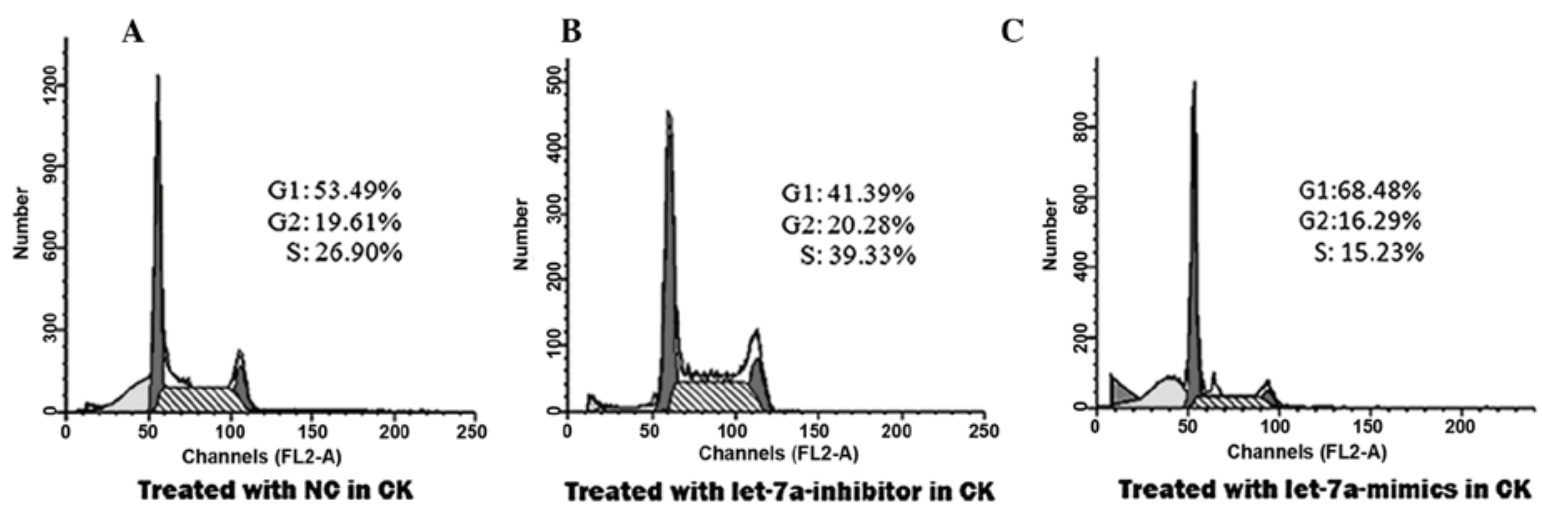

Figure 2. Let-7a inhibits cell proliferation of cholesteatoma keratinocytes (CK) by regulating the cell cycle. Distribution of the cells in the different phases of the cell cycle following transfection of the cholesteatoma keratinocytes with (A) let-7a inhibitor, (B) let-7a mimics or (C) control miRNA (NC).

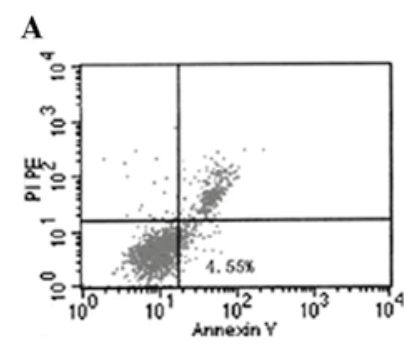

Treated with let-7a-inhibitor in cK

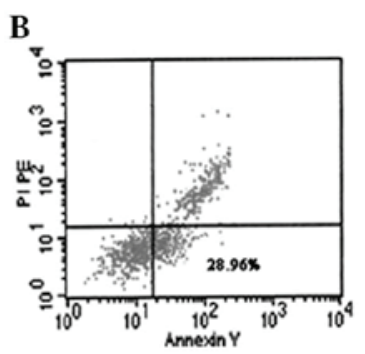

Treated with let-7a-mimies in cK
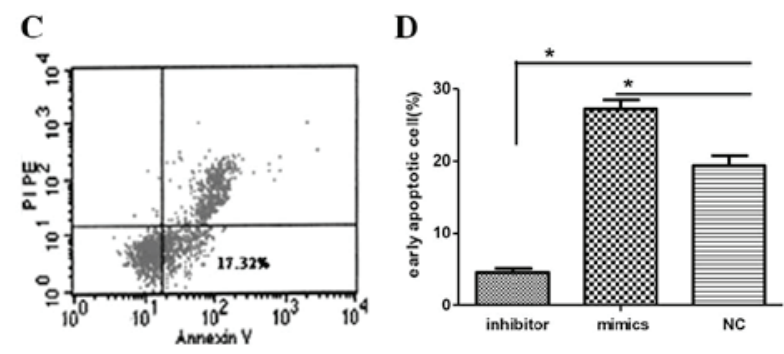

Treated with NC in CK

Figure 3. Let-7a induces early apoptosis of cholesteatoma keratinocytes (CK). Fluorescence-activated cell sorting analysis of the fraction of Annexin V-positive, PIPE-negative cholesteatoma keratinocytes following transfection of the cells with (A) let-7a inhibitor, (B) let-7a mimics or (C) control miRNA (NC). (D) Statistical analysis of the percentage of early apoptotic cells following transfection of the CKs with let-7a inhibitor, let-7a mimics or NC. The data are represented as the mean \pm standard deviation. ${ }^{*} \mathrm{P}<0.05$, Student's t-test. PIPE, propidium iodide and phycoerythrin. 
A

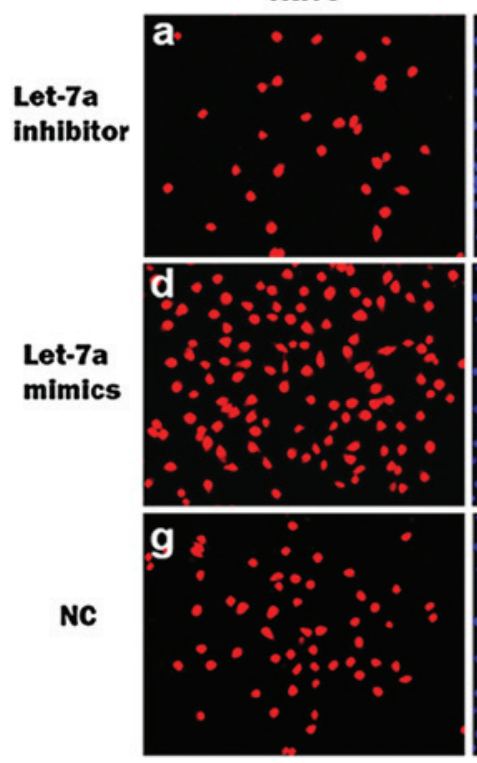

DAPI
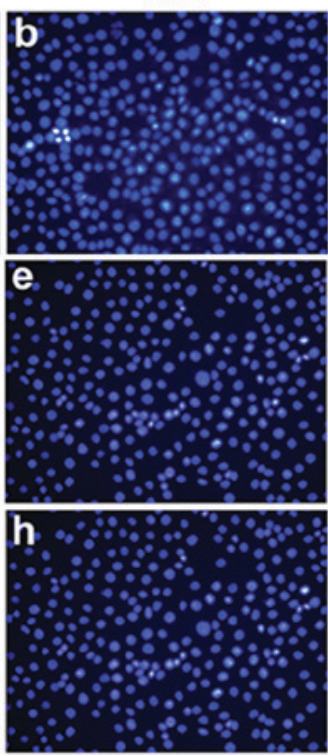

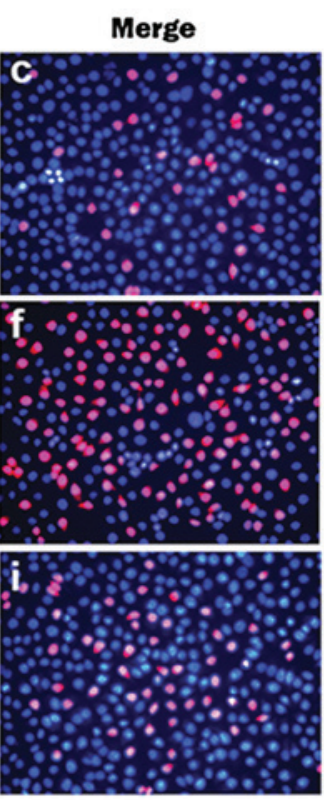

B

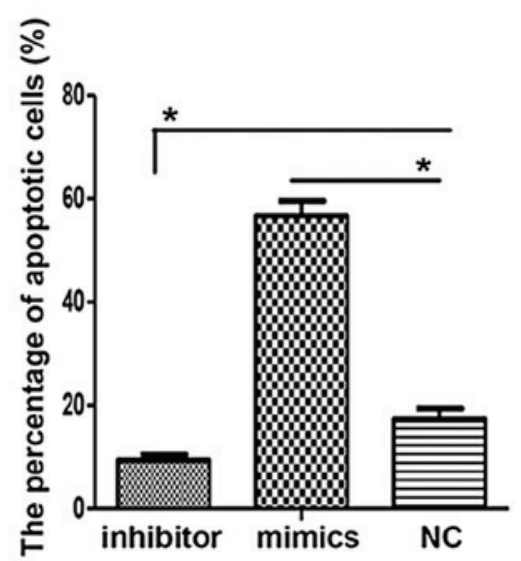

Figure 4. Let-7a promotes cell apoptosis of cholesteatoma keratinocytes. (A) The representative images of terminal deoxynucleotidyl transferase-mediated dUTP-digoxigenin nick end-labeling (TUNEL) label (red; a, d, g), 4',6-diamidino-2-phenylindole (DAPI) staining (blue b, e, h) and their merged images (c, f, i) in cholesteatoma keratinocytes transfected with let-7a inhibitor, let-7a mimics or control miRNA (NC). (B) Statistical analysis of the percentage of apoptotic cells following transfection of the choleasteatoma keratinocytes with let-7a inhibitor, let-7a mimics or NC. Data are represented as the mean \pm standard deviation. ${ }^{*} \mathrm{P}<0.05$, Student's t-test.

A

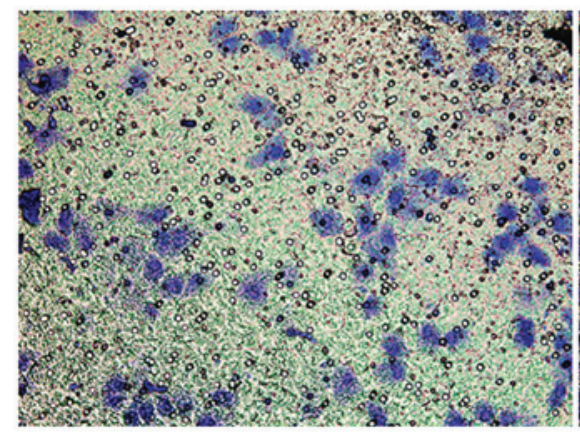

Treated with let-7a-inhibitor
B

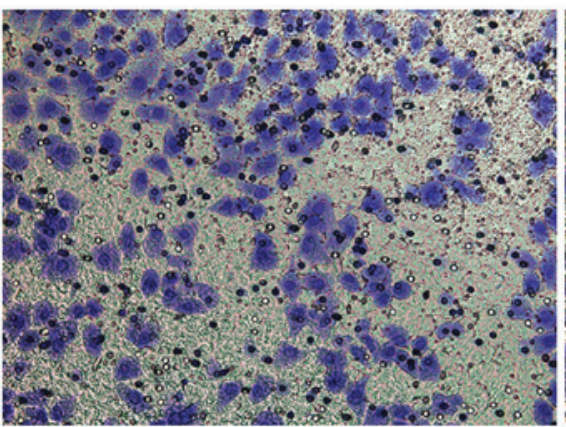

Treated with let-7a-mimics
C

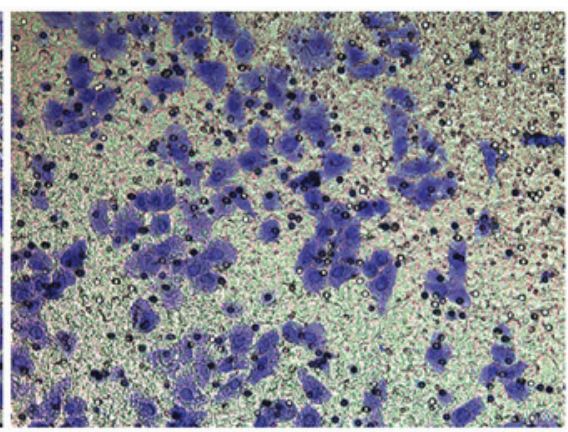

Treated with NC

Figure 5. Let-7a suppresses migration and invasion of cholesteatoma keratinocytes. The representative images of migrated cholesteatoma keratinocytes (KC) (blue) following transfection with (A) let-7a inhibitor, (B) let-7a mimics or (C) control miRNA (NC). Hexamethylpararosaniline staining (blue) was used.

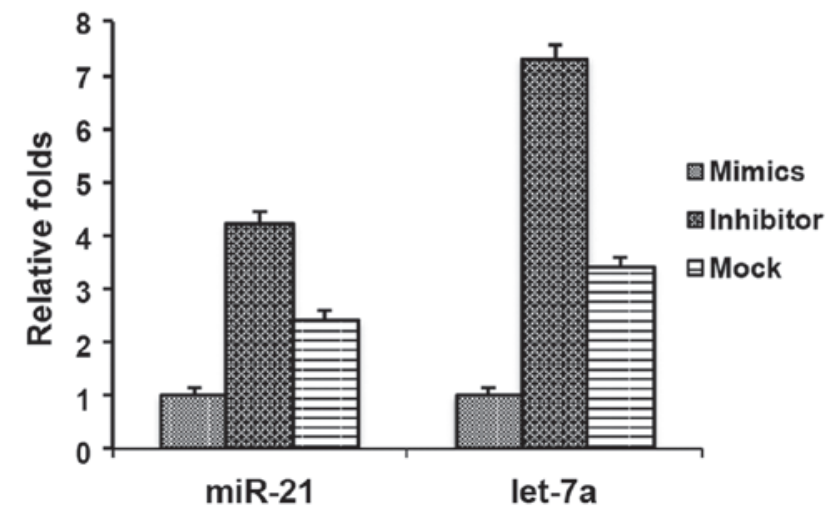

Figure 6. Let-7a downregulates the expression of microRNA (miR)-21. A reverse transcription-quantiative polymerase chain reaction was performed to analyze the expression levels of miR-21 and let-7a in cholesteatoma keratinocytes following transfection with let-7a inhibitor, let-7a mimics or control miRNA. mimics and an upregulation of miR-21 in the cholesteatoma keratinocytes transfected with the let-7a inhibitor (Fig. 6). As a control, the expression of let-7a was determined and shown to be downregulated and upregulated following transfection with let-7a mimics and inhibitor, respectively (Fig. 6). These data suggest that let-7a may regulate miR-21, resulting in the effects on cell proliferation and cell apoptosis.

\section{Discussion}

Previous studies of cholesteatoma growth and proliferation have mainly focused on growth factors and cytokines (2). Growth factors and their receptors, such as EGF/EGFR and $\mathrm{KGF} / \mathrm{KGFR}$, are highly upregulated and associated with cell proliferation in cholesteatomas (3-9). In addition, cytokines including interleukin (IL)-1, IL-6 and tumor necrosis factor- $\alpha$ 
have been shown to be overexpressed and have important roles in the proliferation of cholesteatoma keratinocytes (14-18). However, the roles of miRNAs, which are important regulators of protein translation, have yet to be explored. In previous studies, miRNAs let-7a and miR-21 have been implicated in regulating the proliferation and apoptosis of cholesteatomas $(10,11)$; however, there is currently no direct evidence supporting this. Let-7 is a member of the tumor-suppressing miRNA family and its expression is limited in the majority of human malignancies (19). High expression levels of let-7 have been shown to have antiproliferative effects on cancer cells (20). In head and neck squamous cell carcinoma, it has been reported that low expression levels of let-7d are a prognostic factor for poor survival (21). In numerous tumors of solid organs, let-7a is downregulated $(22,23)$, and acts as a tumor suppressor by targeting oncogenes, including RAS and HMGA2 (24). The present study was the first, to the best of our knowledge, to provide solid evidence to support the roles of let-7a against the growth of cholesteatoma keratinocytes. Let-7a inhibited the proliferation of cholesteatoma keratinocytes by promoting cell cycle arrest in the G0/G1 phase. Furthermore, let-7a induced early and late apoptosis in cholesteatoma keratinocytes. Through its dual roles in inhibiting proliferation and inducing apoptosis, let-7a was shown to be capable of suppressing the growth of cholesteatoma keratinocytes. These findings are concordant with the previous identification of let-7a as a tumor suppressor in other cancer cells $(20,24)$. Notably, the present study identified a novel role of let-7a in preventing migration and invasion of cholesteatoma keratinocytes. Therefore, it may be worth examining whether let-7a has similar functions in the inhibition of migration and invasion of cancer cells in other tumor tissues.

Considering the crucial roles of let-7a in the pathogenesis of cholesteatoma, it would be beneficial to identify the downstream targets of let-7a. miR-21, another miRNA, has previously been shown to be aberrantly overexpressed in cholesteatomas, and to be involved in proliferation, apoptosis and cell growth $(10,11)$. The present study showed that overexpression of let-7a mimics inhibited the expression of miR-21. Conversely, inhibition of let-7a promoted the expression of miR-21. The downstream targets of miR-21, PTEN and PDCD4, have previously been found to be decreased in cholesteatomas, as compared with normal tissues $(10,11)$. Since PTEN and PDCD4 have roles in the inhibition of proliferation and induction of apoptosis in cancer cells $(25,26)$, it may be reasonable to speculate that let-7a initially downregulates the expression of miR-21. Downregulation of miR-21 may then induce the expression of PTEN and PDCD4, resulting in the inhibition of proliferation and induction of apoptosis in cholesteatomas. The present study revealed that let-7a regulation of proliferation and apoptosis may be through controlling the expression of miR-21; however, the downstream effectors that mediate the role of let-7a on migration and invasion remain unknown. This question may be addressed in a further study.

In conclusion, the present study revealed the essential roles of let-7a in inhibiting growth and invasion of cholesteatoma keratinocytes. Furthermore, a potential mechanism was identified, let-7a may regulate miR-21 to control proliferation and apoptosis of cholesteatoma keratinocytes. These findings indicate that let-7a is a pivotal regulator of the pathogenesis of cholesteatomas.

\section{Acknowledgements}

The authors of the present study would like to thank all other members of the laboratory for their technical support and helpful discussion.

\section{References}

1. Louw L: Acquired cholesteatoma: summary of the cascade of molecular events. J Laryngol Otol 127: 542-549, 2013.

2. Preciado DA: Biology of cholesteatoma: special considerations in pediatric patients. Int J Pediatr Otorhinolaryngol 76: 319-321, 2012.

3. Alves AL, Pereira CS, Carvalho Mde F, Fregnani JH and Ribeiro FQ: EGFR expression in acquired middle ear cholesteatoma in children and adults. Eur J Pediatr 171: 307-310, 2012.

4. Barbara M, Raffa S, Murè C, et al: Keratinocyte growth factor receptor (KGF-R) in cholesteatoma tissue. Acta Otolaryngol 128: 360-364, 2008

5. Jin BJ, Min HJ, Jeong JH, Park CW and Lee SH: Expression of EGFR and microvessel density in middle ear cholesteatoma. Clin Exp Otorhinolaryngol 4: 67-71, 2011.

6. Yamamoto-Fukuda T, Takahashi H and Koji T: Expression of keratinocyte growth factor (KGF) and its receptor in a middle-ear cavity problem. Int J Pediatr Otorhinolaryngol 76: 76-81, 2012.

7. Kuczkowski J, Bakowska A, Pawelczyk T, Narozny W and Mikaszewski B: Cell cycle inhibitory protein p27 in human middle ear cholesteatoma. ORL J Otorhinolaryngol Relat Spec 68: 296-301, 2006

8. Liu W, Ren H, Ren J, et al: The role of EGFR/PI3K/Akt/cyclinD1 signaling pathway in acquired middle ear cholesteatoma. Mediators Inflamm 2013: 651207, 2013.

9. Sakamoto T, Kondo K, Yamasoba T, et al: Overexpression of ErbB-2 protein in human middle ear cholesteatomas. Laryngoscope 114: 1988-1991, 2004.

10. Chen X and Qin Z: Post-transcriptional regulation by microrna-21 and let-7a microRNA in paediatric cholesteatoma. J Int Med Res 39: 2110-2118, 2011.

11. Friedland DR, Eernisse R, Erbe C, Gupta N and Cioffi JA: Cholesteatoma growth and proliferation: posttranscriptional regulation by microRNA-21. Otol Neurotol 30: 998-1005, 2009.

12. Djuranovic S, Nahvi A and Green R: A parsimonious model for gene regulation by miRNAs. Science 331: 550-553, 2011.

13. Bartel DP: MicroRNAs: genomics, biogenesis, mechanism, and function. Cell 116: 281-297, 2004.

14. Akimoto R, Pawankar R, Yagi T and Baba S: Acquired and congenital cholesteatoma: determination of tumor necrosis factor-alpha, intercellular adhesion molecule-1, interleukin-1-alpha and lymphocyte functional antigen-1 in the inflammatory process. ORL J Otorhinolaryngol Relat Spec 62: 257-265, 2000.

15. Bujía J, Kim C, Boyle D, et al: Quantitative analysis of interleukin-1-alpha gene expression in middle ear cholesteatoma. Laryngoscope 106: 217-220, 1996.

16. Bujia J, Kim C, Ostos P, et al: Interleukin 1 (IL-1) and IL-1-receptor antagonist (IL-1-RA) in middle ear cholesteatoma: an analysis of protein production and biological activity. Eur Arch Otorhinolaryngol 253: 252-255, 1996.

17. Kato A, Ohashi Y, Masamoto T, et al: Interleukin-6 and tumour necrosis factor alpha synthesized by cholesteatoma cells affect mucociliary function in the eustachian tube. Acta Otolaryngol 38 (Suppl 5): 90-97, 1998.

18. Mehta D, Daudia A, Birchall JP and Banerjee AR: The localization of matrix metalloproteinases- 8 and -13 in cholesteatoma, deep-meatal and post-auricular skin: a comparative analysis. Acta Otolaryngol 127: 138-142, 2007.

19. Thornton JE and Gregory RI: How does Lin 28 let-7 control development and disease? Trends Cell Biol 22: 474-482, 2012.

20. Wong TS, Man OY, Tsang CM, et al: MicroRNA let-7 suppresses nasopharyngeal carcinoma cells proliferation through downregulating c-Myc expression. J Cancer Res Clin Oncol 137: 415-422, 2011. 
21. Childs G, Fazzari M, Kung G, et al: Low-level expression of microRNAs let-7d and miR-205 are prognostic markers of head and neck squamous cell carcinoma. Am J Pathol 174: 736-745, 2009.

22. Mayr C, Hemann MT and Bartel DP: Disrupting the pairing between let-7 and Hmga2 enhances oncogenic transformation. Science 315: 1576-1579, 2007.

23. Johnson SM, Grosshans H, Shingara J, et al: RAS is regulated by the let-7 microRNA family. Cell 120: 635-647, 2005.

24. Park SM, Shell S, Radjabi AR, et al: Let-7 prevents early cancer progression by suppressing expression of the embryonic gene HMGA2. Cell Cycle 6: 2585-2590, 2007.
25. Frankel LB, Christoffersen NR, Jacobsen A, et al: Programmed cell death 4 (PDCD4) is an important functional target of the microRNA miR-21 in breast cancer cells. J Biol Chem 283: 1026-1033, 2008.

26. Zhao H, Dupont J, Yakar S, Karas M and LeRoith D: PTEN inhibits cell proliferation and induces apoptosis by downregulating cell surface IGF-IR expression in prostate cancer cells. Oncogene 23: 786-794, 2004. 